\title{
The Influence of Inclusive Leadership on Employee's Job Performance-A Moderated Mediating Model
}

\author{
Yanru Wang ${ }^{1}$, Yanni Zhu ${ }^{2, *}$, Juan Tang ${ }^{3}$ \\ ${ }^{1}$ Faculty of Hospitality and Tourism Management, Macau University of Science and Technology, Macau, China \\ ${ }^{2}$ Faculty of Hospitality and Tourism Management, Macau University of Science and Technology, Macau, China \\ ${ }^{3}$ Faculty of Hospitality and Tourism Management, Macau University of Science and Technology, Macau, China \\ *Corresponding author. Email: ynzhu@must.edu.mo
}

\begin{abstract}
Drawing on the social exchange theory, this paper discusses the motivational influence of inclusive leadership on job performance. Psychological empowerment was selected as a mediating variable and promotion focus was used as a boundary condition for analysis. Using questionnaires to analyze 416 pairs of leadership-employee data, we found that: 1. Inclusive leadership has a positive impact on psychological empowerment; 2. Psychological empowerment plays a part of the mediating role between inclusive leadership and job performance 3 . Promoting focus has a moderating effect on the job performance of inclusive leaders through psychological empowerment.
\end{abstract}

Keywords: Inclusive leadership, Psychological empowerment, Regulatory focus, Promotion focus, Job performance.

\section{包容型领导对员工工作绩效的影响一一个有调节的 中介模型 \\ 王嫣茹 ${ }^{1}$, 朱燕妮 ${ }^{2, *}$, 唐娟 ${ }^{3}$}

${ }^{1}$ 澳门科技大學酒店与旅游管理学院, 澳门, 中国

2 澳门科技大學酒店与旅游管理学院, 澳门, 中国

澳门科技大學酒店与旅游管理学院, 澳门, 中国

*通讯作者. 邮箱: ynzhu@must. edu. mo

\section{中文摘要}

本文基于社会交换理论，探讨包容型领导对員工工作绩效的动机影响及其边界条件。本文选取心理授权作为中 介变量以及促进定向作为边界条件构建模型, 運用问卷调查法通过对 416 对领导一員工配对数据進行分析发现: 1. 包容型领导对心理授权有正向的影响; 2. 心理授权在包容型领导与工作绩效之间起到部分中介作用; 3 . 促进定 向对包容型领导通过心理授权对工作绩效的影响有调节作用。

关键词: 包容型领导; 心理授权; 调节定向理论; 促进定向; 工作绩效

\section{1. 引言}

随著经济的不断增长, 旅游业成为第三产业中的 重要组成部分 ${ }^{[1]}$, 是世界上发展最快的新兴产业之 一。旅游行业的日益崛起, 使酒店行业的竞争也逐 渐加剧, 为了更好地适应国际化进程, 酒店面对著不
同民族、不同信仰、不同价值观以及不同文化的员 工。因此需要酒店企业在组织的管理当中具有更高 的包容性。当领导者可以改变或者影响被领导者的 思想或行为, 那麼领导者就可以很容易发挥他自身 的领导功能, 充分调动被领导者在工作中的积极性 与创造性。 
包容型领导是一种善于听取员工意见和观点并且 从言行上鼓励和欣赏员工的一种领导方式 ${ }^{[2]}$, 是一 种组织或团队中善于倾听和关注员工的需要。为了 表现员工在多大程度接受包容型领导进而影响员工 的工作绩效，本文引入了心理授权这个概念。心理 授权体现了一种自我表达的内在的工作动机 ${ }^{[3]}$, 是 被授权的个体内心体验的综合体。包容型领导包容 员工独特的看法和见解 ${ }^{[4]}$, 鼓励员工自我决策, 领 导对员工表现出信任, 增强了员工的自我效能感 ${ }^{[5]}$, 从而会提升员工的心理授权 ${ }^{[6]}$ 。促进定向(promotion focus)体现了个体的自我调节方向或倾向,强调个人 的成果、成就和为了追求更大的收穫。李燕萍指出, 在信息技术高速发展的背景下,新生代员工（80、90 后出生）逐渐成为职场的主力军 ${ }^{[7]}$, 他们渴望成功, 希望在工作中得到认可。已有研究表明, 领导行为 可以影响员工的调节定向 ${ }^{[8]}$ 。当领导强调个人成果、 成就, 追求成功时, 可以激发员工促进定向的产生。 基于自我调节,员工会通过改变自己的心理或行为以 适应领导的管理方式 ${ }^{[9]}$ 。综上所述, 本文将尝试以 社会交换理论为框架, 以心理授权为中介变量, 探 讨包容型领导力对员工工作绩效的影响机制, 并引 入促进定向作为模型的调节变量。

\section{2. 研究理论和研究假设}

\section{1. 研究理论}

本研究将以社会交换理论为框架, 探讨包容型领 导与工作绩效的内在影响机制以及形成这种关系的 边界条件。根据社会交换理论 ${ }^{[10]}$, 当领导向员工提 供非物质或物质资源时, 会与员工形成社会交换关 系, 使员工产生回馈的意愿与行动。根据社会交换 理论中的互惠原则 ${ }^{[11]}$, 领导给予员工较多的信任和 支持, 就会使员工增强对组织的忠诚度来回应与组 织之间平衡互惠的交换关系 ${ }^{[12]}$ 。Organ 和 Konovsky ${ }^{[13]}$ 提出员工和组织间的交换是一种经济与 社会的混合物, 他们强调频繁表现出的角色外行为, 也反映出与组织的关系主要是一种社会交换关系, 并不是合同固有的义务。当员工在组织中获得资源 后，会产生一种回报组织的责任意识。

\section{2. 心理授权的中介作用}

Nembhard 和 Edmondson ${ }^{[2]}$ 首次将“包容”引入到组 织的管理当中，并提出了“包容型领导”这个概念,他 们指出包容型领导是指领导欣赏并认可员工在工作 中所做出的贡献。包容型领导从人的本质出发, 首 先关注到的是个体所具有的独特性, 进而增强员工 在组织中的归属感 ${ }^{[14]}$ 。员工的独特性是指他们保持 独特的自我意识的需要 ${ }^{[15]}$, 而员工的归属感体现在 发展或保持稳固的人际关系的需要 ${ }^{[16]}$, 两者都需得 到满足才能使员工感受到包容的存在。Carmeli 等人 [17]提出包容型领导在与员工互动的过程中表现出的 特性是开放性、可用性和易接近性, 开放性指领导
乐意听取员工意见; 可用性指领导可以为员工提供 专业领域的意见; 易接近性指领导主动关心自己的 员工。包容型领导被运用在组织管理层面, 被证明 领导的包容型对员工或者团队的绩效有显著影响 ${ }^{[18}$ 21]。

心理授权被定义为一个综合的情感过程, 它体现 了一种自我表达内在的工作动机 ${ }^{[3]}$, 心理上被授权 的员工能够胜任自己的工作, 表现出自我决定的取 向, 认为自己的工作是有意义并且可以获得影响的 [22 24], 后来心理授权概念所关注的是员工对授权的 认知, 这种认知会控制他对工作的动机, 它包含了 四个维度：工作意义, 自我效能感, 决策自主性以 及影响力 ${ }^{[17]}$ 。工作意义指个体对根据自己的标准判 断工作目标的重要性的认知 ${ }^{[24]}$, 自我效能感指个体 对自我是否能完成某项任务的认知 ${ }^{[25]}$, 决策自主性 指个体对自己的工作是否具有控制权 ${ }^{[26]}$, 影响力指 个体对组织管理的影响程度 ${ }^{[27]}$ 。心理授权作为一种 主观感知既会对员工工作的内在动机产生影响, 也 会受到领导风格的影响 ${ }^{[6]}$ 。

包容型领导对心理授权的逻辑具体表现在包容型 领导对心理授权的四个维度分别产生了积极的影响: 第一，工作意义方面。包容型领导与其他领导风格 不同，具有这种领导风格的领导肯定员工的贡献， 关注员工的需求 ${ }^{[2]}$, 这种行为会提升员工的幸福感 和工作满意度 ${ }^{[28]}$, 根据工作中的要求与自身价值观 及行为准则来评估自己的工作意义, 让员工在工作 中明白自身的价值, 以此增加了工作的信心和动力, 继而增加员工的心理授权程度。第二，自我效能感 方面。包容型领导增加了员工试错的勇气和机会, 促使员工不断创新进步。合理运用每一个员工自身 的优势, 提高员工工作的信心和动力, 这样不仅可 以提高员工的自我效能 ${ }^{[5]}$, 也可以提高员工的心理 授权程度 ${ }^{[6]}$ 。第三, 决策自主性方面.Deci 和 Ryan ${ }^{[29]}$ 的自我决定理论认为环境可以通过自主、胜任、关 系这三方面满足人的内心需求增强内部动机 Hollander ${ }^{[30]}$ 认为包容型领导与下属之间是一种双向 关系，领导与下属之间共同完成任务,实现目标双赢. 决策不应该只取决于领导，还应该采纳员工的意见， 领导的包容性使员工拥有决策自主性, 满足员工内 在需求从而提高心理授权。第四, 影响力方面。员 工参与到组织的活动中, 为组织出谋划策, 对组织 的发展产生影响, 领导就会认可员工的贡献, 员工 对组织产生影响力从而增加心理授权程度。综上, 提出如下假设: 系。

$\mathrm{H} 1$ ：包容型领导与员工的心理授权呈现正相关关

Motowildlo ${ }^{[31]}$ 提出绩效是个体在标准时间内进行 的离散行为发作的组织的总期望值。Wayne ${ }^{[32]}$ 指出, 领导者通过表现出热情, 可以鼓励被领导者的自尊 心, 从而激发被领导者工作的积极态度。社会交换 理论认为, 人们在社会交换的这个过程中遵循互利 
共赢的原则。当员工的经济和情感需求在组织中得 到满足，他们就会产生对组织认同的责任意识,“员工 的高工作满意程度会提升员工的士气, 而较高的士 气会促进生产效率的提高”[33]。包容型领导的开放性 拉近了领导和员工之间的距离。领导能够认真听取 员工意见并且可以及时有效的对员工所做的贡献进 行反馈, 体现了员工在工作中可以自由言论的权利。 这不仅增强了员工在工作责任感也增加了员工对组 织的忠诚度, 从而能让员工发挥更大的潜能创造更 出色的业绩。包容型领导关注员工的独特性, 每个员 工都是独立的个体, 领导要在工作中重视员工之间 的独特性, 发掘他们的潜能, 进一步来看, 本文认 为当员工感受到较高的心理授权时, 工作绩效也会 随之提高,第一, 已有研究表明, 员工对领导的包容 程度的感知与其心理安全认知水准呈正相关 ${ }^{[17]}$, 包 容型领导作为关系型领导的具体形式, 它更注重领 导与员工之间的关系, 也更关心员工的心理需求, 增强员工的归属感从而提高工作绩效。第二, 根据 Deci 和 Ryan ${ }^{[27]}$ 的自我决定理论中提到个体在充分认 识到自己所需要的环境的基础上, 对自己的行为作 出的选择。当包容型领导足够充分时, 开放的氛围 会影响员工的心理授权，自我效能感是心理授权的 组成部分, 被证明与绩效有关 ${ }^{[34]}$ 。第三, 领导对员 工提供包容的氛围, 当员工意识到在工作中的意义 时, 他们会专注于自己的工作, 这也会提高工作绩 效 ${ }^{[35 ~ 36]}$. 因此, 当包容型领导氛围足够时, 会提高员 工的心理授权, 进一步提高工作绩效; 反之亦然。综 上，提出如下假设：

$\mathrm{H} 2$ : 心理授权在包容型领导与工作绩效之间起到 中介作用。

\section{3. 促进定向的调节作用}

调节定向理论用于瞭解人们追求愉悦或避免痛苦 的动机 ${ }^{[37 \sim 38]}$, 它区分了促进定向(promotion focus)和 预防定向(prevention focus)两种不同的自我调节方向。 促进定向强调个人的成果、成就和为了追求更大的 收穫。随著在资讯技术高速发展时代背景下的新生 代员工 $\left(80 、 90\right.$ 后出生)逐渐成为职场的主力军 ${ }^{[7]}$, 新 生代员专注于晋升时, 渴望创造机会, 并害怕错过 那个潜在机会 ${ }^{[39]}$, 所以本文选取了更加契合新生代 员工特质的促进定向作为边界条件进行研究。

领导者的有效性取决于员工的自我调节倾向, 只 有当追随者愿意投入自己的能力时, 这种有效性才 会出现 ${ }^{[40]}$ 。本研究认为, 包容型领导所提供的氛围 可以使具有促进定向员工的内在得到满足，从而提 高员工的心理授权。促进定向的调解作用表现在:第 一, 由于调节定向本身是个体的特质 ${ }^{[41]}$, 会受到外 部环境影响产生的短暂性调节定向 ${ }^{[42]}$, Spritzer ${ }^{[23]}$ 指 出授权水准高低受到自我控制和自尊心的影响, 自 我控制倾向高的员工完成工作的主动性也强。因此, 具有促进定向的员工在追求成功的过程中受包容型
领导的开放性氛围影响对工作表现出主动性, 自我 控制能力较强, 从而提升心理授权。第二, Crowe 和 Higgins ${ }^{[43]}$ 在研究中发现, 当不得不提出许多可能 的替代想法时, 以晋升为重点的人会产生更多不同 的想法。进一步的研究发现, 以促进为重点的人比以 预防为重点的人更擅长创造性思维 ${ }^{[44]}$, 并且更容易 识别或创造机会 ${ }^{[4]}$ 。第三, 具有强烈促进定向的员 工能够提升综合思维能力, 迸发事物间微妙的联系 [45]。据此, 本文认为促进定向有助于提升自我效能 感，具有促进定向的员工在包容的氛围下可以更好 的完成工作任务, 从而提高心理授权。也就是说,具 有促进定向的员工对领导所提供的包容行为更加敏 感, 当领导表现出对员工的尊重和重视时, 员工更 容易表达自己追求成功的意愿, 内心得到满足, 心 理授权程度也会相应提高。综上, 提出如下假设:

H3：促进定向正向调节了包容型领导对员工心理 授权的影响。

结合假设 2 和假设 3 , 本文认为促进定向可以强 化心理授权在包容型领导与工作绩效之间的中介作 用: 第一, 经典动机理论认为, 人们对成功的高期 望会引发高动机, 从而会促进积极结果的产生 ${ }^{[49]}$, 员 工在理想框架下会产生促进定向动机，继而更倾向 于理想自我的目标诱导 ${ }^{[38]}$ 。研究表明, 怀有强烈促 进定向的员工对新成果十分敏感, 并重视获得成功 的相关信息, 因此他们做事更加精益求精 ${ }^{[46]}$ 。具有 促进定向的员工在领导的包容性下，他们追求更好 的目标获得心理授权就可以创造出更高的工作绩效。 第二, 员工对采集到的信息经过整合赋予全新的理 解, 该理解会最终推动一系列的创意形成 ${ }^{[48]}$ 。当促 进定向的员工面对棘手的问题时，他们擅长奇思妙 想并且广泛尝试的特点会提高成功的可能性，带来 工作成果 ${ }^{[49]}$ 。具有促进定向的员工可以在领导提供 的包容性氛围下敢想、敢说、敢做, 提升心理授权 程度从而提工作绩效。第三, 结合调节定向匹配理 论，当领导者的某方面行为与员工的调节定向相呼 应时, 员工对领导的重视才会发生 ${ }^{[50]}$ 。当具有促进 定向的员工与领导所提供的包容性氛围相呼应,员工 就会重视自己的工作增加自我效能感从而增加心理 授权程度, 在工作中最求更加积极的结果进一步提 高工作绩效。综上，提出如下假设：

H4: 员工的促进定向正向调节了包容型领导通过 心理授权为中介对员工工作绩效的影响。

本研究建构模型如图 1 所示:

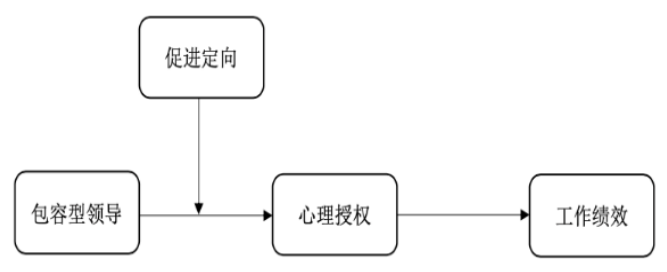

图 1 本研究模型图 


\section{3. 研究方法}

\section{1. 研究方法和程序}

本研究的调查对象是酒店行业的专业团队，通过 纸质问卷的形式发送。研究者将问卷分为三个阶段 行发放,第一阶段为 2019 年 8 月 19 日至 2019 年 9 月 9 日，第二阶段为 2019 年 9 月 26 日至 2019 年 10 月 14 日，第三阶段为 2019 年 10 月 21 日至 2019 年 11 月 1 日。每个阶段发放 500 份问卷。为了确保三个 阶段发放的问卷成功匹配, 研究者设计了两道配对 问题: “您的员工工号”以及“您联系方式的后四位”, 每位参与者必须在三个时间点所填的问卷上回答这 两道问题, 以便研究者后续配对问卷。

在回收三个阶段的问卷后, 成功配对且有效的问 卷共 416 份, 回收率为 $83.2 \%$ 。在最终结果中, 女 性占 $55.3 \%$; 年龄集中在 $18-25$ 岁, 占 $41.3 \%$; 教育 程度在高职高专、本科人数各占 $29.6 \%$ 。

\section{2. 变量测量}

问卷中所涉及的所有题项均直接采用国外现已发 展成熟的相应量表。由于此次问卷发放地点在中国 大陆, 因此遵循标准的翻译和回译（translation and back translation) 程式, 把所有的英文测量工具准确 地翻译为中文 ${ }^{[51]}$ 。本次测量均采用 Likert 5 点量表 ( $5=$ 非常同意; $1=$ 非常不同意) 。员工需要评价领 导的包容性, 以及自评促进定向和心理授权的认同 度。此外, 领导需要对自己部门员工的工作绩效进 行评估。

（1）包容型领导: 采用 Carmeli, A. 等 ${ }^{[17]}$ 编制的 量表。包括: “我的主管乐意听取我提出的新方案” 在内等 9 个题项。

（2）心理授权: 采用 Spreitzer, G. ${ }^{[23]}$ 编制的量表。 包括: “我所做的工作对我来说非常重要”在内等 12 个题项。

（3）促进定向：采用 Lockwood, P. 等 ${ }^{[52]}$ 编制的 量表。其中，促进定向包括:“我经常想像我将如何 实现我的希望和抱负”在内等 12 个题项。

（4）工作绩效: 采用 Kenneth S. Law, 等 ${ }^{[33]}$ 编制 的量表, 包括: “他/她有很大的工作兴趣和热情”在内 等 6 个题项。

(5) 控制变量: 在本研究中, 控制了员工的性 别,年龄及教育程度。

本研究使用 Andrew F. Hayes（2013）开发的基 于 SPSS 和 SAS 的 process 插件进行中介效应分析; 使用 AMOS 26.0 对数据进行效度分析。

\section{4. 研究结果与分析}

\section{1. 相关系数分析}

从表 1 呈现的数据来看, 相关系数的结果与研究 者预期的效果方向基本一致，也使得研究模型得到 了初步的支持。

\section{2. 验证性因子分析}

信度。由于本研究所采用量表为前人编制, 包容 型领导量表的 Cronbach's $\alpha$ 为 $0.94^{[17]}$; 心理授权量 表的 Cronbach's $\alpha$ 为 $0.88^{[23]}$; 促进定向量表的 Cronbach's $\alpha$ 为 $0.81^{[52]}$; 工作绩效量表的 Cronbach's $\alpha$ 为 $0.91^{[53]}$ 。变量值大于 0.8 , 均在可接受范围内。

效度。从表 2 中可以看出, $\mathrm{X} 2 / \mathrm{df}$ 的值为 1.2 , 小 于 3 ; 结果配适理想, RESEA 为 0.02 , 小于 0.05 ,结 果配适理想; GFI、AGFI、RFI、IFI、TLI 的结果均 大于 0.9 , 结果配适良好。意味著本次分析的数据具 有良好的结构效度。

表 2 结构效度（N=416）

\begin{tabular}{lllllll}
\hline $\mathrm{X}^{2} / \mathrm{df}$ & RESEA & GFI & AGFI & RFI & IFI & TLI \\
\hline 1.2 & 0.02 & 0.92 & 0.91 & 0.93 & 0.99 & 0.99 \\
\hline
\end{tabular}

从表 3 中可以看出, 四个变量对应的 AVE 值均 都大于 0.5 , 且 $C R$ 值全部都高于 0.7 , 意味著本次 分析数据具有良好的聚合效度。

表 3 聚合效度

\begin{tabular}{ccc}
\hline 因素 & $\begin{array}{c}\text { 平方差萃取 } \\
\text { AVE 值 }\end{array}$ & $\begin{array}{c}\text { 组合信度 CR } \\
\text { 值 }\end{array}$ \\
\hline 包容型领导 & 0.58 & 0.81 \\
心理授权 & 0.58 & 0.85 \\
促进定向 & 0.53 & 0.77 \\
工作绩效 & 0.61 & 0.76 \\
\hline
\end{tabular}

从表 4 中可以看出, 各变量之间都具有显著性 $(\mathrm{P}<0.01)$, 且低于 AVE 的平方根值, 说明本次分析 数据具有良好的区分效度。

表 1 均值、标准差以及变量之间的相关系数

\begin{tabular}{|c|c|c|c|c|c|c|c|c|c|}
\hline & M & SD & 性别 & 年龄 & 教育程度 & 包容型领导 & 促进定向 & 心理授權 & 工作颉效 \\
\hline 性别 & 1.55 & 0.50 & & & & & & & \\
\hline 年龄 & 2.08 & 1.16 & 0.09 & & & & & & \\
\hline 教育程度 & 3.08 & 1.25 & -0.07 & $-0.17 * *$ & & & & & \\
\hline 包容型领导 & 3.67 & 0.82 & 0.06 & 0.04 & 0.05 & (0.94) & & & \\
\hline 促进定向 & 3.68 & 0.98 & 0.05 & 0.04 & -0.08 & $0.15^{* *}$ & $(0.81)$ & & \\
\hline 心理授權 & 3.69 & 0.93 & 0.04 & -0.01 & 0.06 & $0.46^{* *}$ & $0.31 * *$ & $(0.88)$ & \\
\hline 工作绩效 & 3.88 & 0.82 & -0.02 & 0.02 & 0.01 & $0.51^{* *}$ & $0.19 * *$ & $0.55^{* *}$ & $(0.91)$ \\
\hline
\end{tabular}

注: 样本量 $\mathrm{N}=416$; 对性别来说, $1=$ 男性, $2=$ 女性; 对年龄来说, $1=18-25$ 岁, $2=26-30$ 岁, $3=31-40$ 岁, $4=41-50$ 岁, $5=51-60$ 岁, $6=60$ 岁以上; 对教育程度来说, $1=$ 初中毕业及以下, $2=$ 高中毕业, $3=$ 高职、高专, $4=$ 本科, $5=$ 硕士及以上学历。括弧内为该量表之 Cronbach's $\alpha, * * \mathrm{p}<0.01$ 
表 4 区分效度

\begin{tabular}{ccccc}
\hline & 包容型领导 & 心理授权 & 促进定向 & 工作绩效 \\
\hline 包容型领导 & $(0.58)$ & & & \\
心理授权 & $0.60^{* *}$ & $(0.58)$ & & \\
促进定向 & $0.65^{* *}$ & $0.35^{* *}$ & $(0.53)$ & \\
工作绩效 & $0.22^{* *}$ & $0.33^{* *}$ & $0.27^{* *}$ & $(0.61)$ \\
AVE 平方根 & 0.76 & 0.76 & 0.73 & 0.78 \\
\hline
\end{tabular}

注: 括号内为该变量 $\mathrm{AVE}$ 的平方根, $* * \mathrm{p}<0.01$

\section{3. 假设检验}

中介效应检验。假设一提到包容型领导与员工的 心理授权呈现正相关关系。从表 5 中可以看出, 包 容型领导对心理授权的直接效应为 0.41 , 置信区间 为 $[0.33,0.50]$, 信区间内不包含 0 , 说明包容型领 导与心理授权之间具有正相关关系，假设一得到了 支持。

假设二提到心理授权在包容型领导与工作绩效之 间有中介作用。根据表 5 , 包容型领导对工作绩效 的直接效应及心理授权的中介效应的置信区间内不 包含 0 , 表明包容型领导可以直接预测工作绩效, 并根据 Baron 和 Kenny ${ }^{[54]}$ 方法可知心理授权在包容 型领导与工作绩效之间起到部分中介作用, 假设二 得到支持。

调节效应检验。假设三提到了促进定向正向调节 了包容型领导对员工心理授权的影响。由表 5 可以 看出, 包容型领导*促进定向的交互项对心理授权的 直接效应为 0.12 , 置信区间为 $[0.04,0.20]$, 置信区 间不包含 0 , 假设三得到支持。

由图 2 可以看出, 当加入心理授权作为中介变量 后, 进一步简单斜率分析表明（图 2)，促进定向 较低(M-1SD)的被试及促进定向较高(M+1SD)的被试, 都展现出包容型领导对心理授权具有显著正向作用。 表明促进定向越高的员工, 包容型领导与心理授权 呈逐渐升高的趋势。此外, 在促进定向的三个水准 上, 心理授权在包容型领导与工作绩效关系中的中 介作用呈上升趋势（见表 6)，即随著促进定向的 提高, 包容型领导会通过心理授权提升员工的工作 绩效，假设四得到支持。

表 5 有调节的中介模型检验

\begin{tabular}{|c|c|c|c|c|c|c|c|c|}
\hline \multirow{3}{*}{ 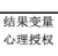 } & \multicolumn{4}{|c|}{ 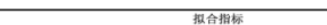 } & & & \multicolumn{2}{|c|}{ 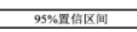 } \\
\hline & 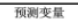 & $\mathrm{R}^{2}$ & $F$ & coeff & $s e$ & $t$ & एटl & ULCL \\
\hline & 性踢 & & & 0.02 & 0.08 & 0.18 & -0.15 & \\
\hline & 年緟 & & & -0.03 & 0.04 & -0.69 & -0.10 & \\
\hline & 整有㭌度 & & & 0.04 & 0.03 & 1.31 & -0.22 & \\
\hline & 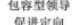 & & & $\begin{array}{l}0.41 \\
0.25\end{array}$ & $\begin{array}{l}0.04 \\
0.05\end{array}$ & $\begin{array}{l}9.77 * * * \\
\text { sag*: }\end{array}$ & $\begin{array}{l}0.33 \\
0.17\end{array}$ & $\begin{array}{l}0.50 \\
0.34\end{array}$ \\
\hline & 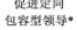 & & & $\begin{array}{l}0.25 \\
0.12\end{array}$ & $\begin{array}{l}0.05 \\
0.04\end{array}$ & $\begin{array}{l}5.94 * * * \\
2.87 *\end{array}$ & $\begin{array}{l}0.17 \\
0.04\end{array}$ & $\begin{array}{l}0.34 \\
0.20\end{array}$ \\
\hline 工性续统 & 绝选定向 & 0.39 & 31.98 & & & & & \\
\hline & 性别 & & & -0.12 & 0.08 & -1.50 & -0.27 & \\
\hline & 年能 & & & 0.01 & 0.03 & 0.15 & $-0,06$ & 0.07 \\
\hline & 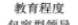 & & & -0.03 & 0.03 & 0.09 & -0.09 & 0.03 \\
\hline & 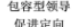 & & & $\begin{array}{l}0.32 \\
0.01\end{array}$ & $\begin{array}{l}0.04 \\
0.04\end{array}$ & $\begin{array}{l}7.26^{\circ} \\
0.28\end{array}$ & $\begin{array}{r}0.24 \\
-0.07\end{array}$ & $\begin{array}{l}0.41 \\
0.09\end{array}$ \\
\hline & 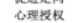 & & & 0.40 & 0.05 & $8.75^{\circ}=$ & 0.31 & \\
\hline & 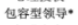 & & & -0.05 & 0.04 & -1.13 & -0.14 & 0.04 \\
\hline & 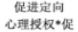 & & & 0.02 & 0.05 & $0.47 * *$ & 0.07 & 0.11 \\
\hline
\end{tabular}

表 6 在促进定向不同水准上的直接效应与中介效应

\begin{tabular}{cccccc}
\hline & 促进定向 & Effect & BoostsSE & BoostsLLCL & BoostsULCL \\
\hline \multirow{4}{*}{ 直接效应 } & effl(M-1SD) & 0.11 & 0.03 & 0.05 & 0.19 \\
& eff2(M) & 0.17 & 0.29 & 0.11 & 0.23 \\
& eff3(M+1SD) & 0.23 & 0.04 & 0.15 & 0.33 \\
& effl(M-1SD) & 0.30 & 0.06 & 0.18 & 0.41 \\
心理报权的中介效应 & eff2(M) & 0.41 & 0.04 & 0.33 & 0.50 \\
& eff3(M+1SD) & 0.53 & 0.06 & 0.41 & 0.65 \\
\hline
\end{tabular}

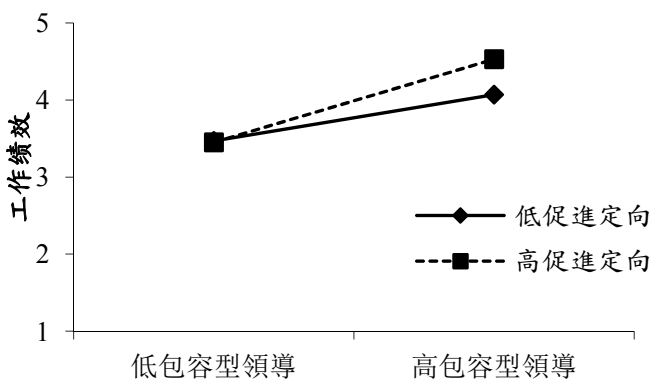

图 2 促进定向对包容型领导-心理授权-工作绩效这 一中介机制的调节作用

\section{5. 讨论与展望}

\section{1. 结果分析}

本研究从心理授权这一视角来研究包容型领导与 工作绩效之间的关系，研究心理授权是如何在这一 关系中起到中介作用, 并探索促进定向作为其边界 调节的影响。

\section{2. 理论贡献}

基于上述结果分析, 本研究的主要贡献有: 首先, 拓展了包容型领导有效性的研究范围。在组织行为 学中, 领导风格作为提高工作绩效的一项核心要素

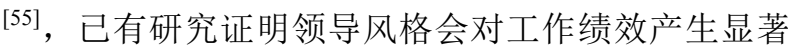
正向影响 $\left.{ }^{[56} 57\right]$, 本文以中国大陆酒店作为研究对象, 将国外学者所开发的关于包容型领导的量表在中国 文化背景下进行测试, 拓展了量表的使用范围。其 次, 丰富了包容型领导的作用机制研究。本研究以 社会交换理论为框架，提出并发现心理授权可以将 包容型领导的正向影响传递到工作绩效。在组织管 理层面，已被证明领导的包容性对员工或者团队的 绩效有显著影响 ${ }^{[18,58-59]}$.Menon ${ }^{[60]}$ 所提出, 个体在心 理授权体验之后, 授权型的领导风格才会有影响作用。 表 5 呈现的数据证明了心理授权在包容型领导与工 作绩效之间起到部分中介作用，表明包容型领导不 仅可以直接影响工作绩效，还会通过心理授权对工 作绩效产生正向影响。本研究结果不仅扩大了包容 型领导的研究范围, 同时也扩展了心理授权的前因 研究.第三，丰富了包容型领导通过心理授权对工作 绩效的边界条件研究。从图 2 中可以看出, 对于高 
促进定向的员工来讲，他们对包容型领导的感知程 度较高, 因此包容型领导的高低对心理授权的影响 会升高, 进而对工作绩效产生正向影响。这一结果 厘清了包容型领导通过心理授权进而影响工作绩效 这一机制的边界条件.

\section{3. 实践意义}

本研究对酒店管理有三方面重要启示。首先, 本 文对包容型领导的实证研究, 充分证明了包容型领 导在中国组织管理中的实现意义, 管理者应该在实 际工作中注意到这一点。因此, 结合包容型领导的 特点, 企业在选用管理人员时, (1) 应该注意到候 选人是否关注到每个员工的特征合理进行人岗匹配;

(2) 候选人是否关注到员工对企业所做出的贡献, 对员工的赞许和包容可以提高工作满意度 ${ }^{[62]}$, 员工 在更好的氛围中工作, 为企业创造更大利润。(3) 候选人是否可以为员工提供专业领域的说明。酒店 业作为人员密集型和准入门槛较低的行业, 员工文 化水平参差不齐, 管理者对员工进行培训, 在工作 中提供说明, 增加员工归属感, 为企业创造更大效 益。其次, 管理者应该关注员工的内在需求, 鼓励 员工参与到公司的决策中, 增强员工的心理授权, 提高工作绩效。最后, 企业要发掘员工的潜质, 给 予他们更多的信任和尊重,增强他们对组织贡献力量 的意愿, 实现目标共赢。

\section{4. 局限性及未来研究方向}

虽然本研究证实了包容型领导与工作绩效的中介 机制及调节效应, 但研究仍然存在一些不足。（1） 本研究的测量题目都采用了李克特五点量表, 题目 大部分为正向叙述, 极容易出现填写类似的情况。 收集到的数据可能会受到同源方差问题的影响 (common method variance, CMV), 为了降低 CMV, 本研究采用程式修正向酒店主管及其下属分别派发 问卷, 降低同源方差对模型的影响。但由于问卷三 次发放的间隔时间较短, 受访者所处环境也会影响 问卷结果,在未来的问卷设计中可以运用程式修正使 问卷更加精益求精. (2) 由于问卷的发放地的局限, 研究结果不能代表全国的情况。因此, 在未来的研 究中可以扩充问卷的发放区域。（3）本研究中不论 是员工对领导的包容性评价、对自身促进定向和心 理授权的自评及领导对员工工作绩效的评价都受到 主观的影响. 虽然处理数据时发现这些偏差来带的影 响并不大, 但仍然值得关注。因此未来对包容型领 导的调查可以增加其调查来源。(4) 本研究只选择 了促进定向作为调节变量进行研究, 但不可忽视具 有预防定向的个体。因此, 在未来的研究中可以加 入预防定向进行研究, 丰富研究结果。

\section{REFERENCES}

[1] Jianjun, S., Gennian, S., Lifang, W. An analysis of China's tourism industry's relevance to the tertiary industry sincee 1982. Progress in Geographical Science, 2011, (8):105-113.

[2] Nembhard, I. M., \& Edmondson, A. C. Making it safe: The effects of leader inclusiveness and professional status on psychological safety and improvement efforts in health care teams. Journal of Organizational Behavior, 2006, 27(7):941-966.

[3] Scott B. D., Christian J. R., \& Mary B. M. Transformational leadership, psychological empowerment, and the moderating role of mechanistic-organic contexts. Journal of Organizational Behavior, 2014, 35: 413-433.

[4] Basharat J., Mehdi R. N., \& Karim K. A., et al. Impact of inclusive leadership on innovative work behavior: The role of psychological safety. Journal of Management \& Organization, 2017, 23(3):1-20.

[5] Chunying, G. The Influence of Inclusive Leadership on Relationship Conflict-Based on the Perspective of Psychological Empowerment and Distribution Justice. Research on Financial Issues, 2016,(6):159-168.

[6] Liu, B., Yan,S., Lei, Q. The Influence of Inclusive Leadership on Relationship Conflict-Based on the Perspective of Psychological Empowerment and Distribution Justice. Research on Financial Issues, 2017, (4):107-113.

[7] Yanping, L., Ting, Y., Yajuan, P. Construction and Implementation of Inclusive Leadership--Based on the management perspective of the new generation of employees. China Human Resources Development, 2012, 3:31-35.

[8] Neubery M. J., Kacmar K. M., \& Carlson P. S., et al. Regulatory focus as a mediator of the influence of initiating structure and servant leadership on employee behavior. Journal of Applied Psychology, 2008, 93(6):1220-1233.

[9] Kark R., \& Van D. D. Motivation to lead, motivation to follow: the role of the self-regulatory focus in leadership processes. Academy of Management Review, 2007, 32:500-528.

[10] Blau P. M. Exchange and Power in social Life. New York: Wiley, 1964.

[11] Cropanzano, R., \& Mitchell, M.S. Social exchange theory: An interdisciplinary review. Journal of Management, 2015, 31(6):874-900.

[12] Avolio, B. J., Weichun, Z., Koh, W. \& Bhatia, P. Transformational Leadership and Organizational 
Commitment: Mediating Role of Psychological Empowerment and Moderating Role of Structural Distance. Journal of Organizational Behavior, 2004, 25(8):951-996.

[13] Organ, D. W., \& Konovsky, M. Cognitive versus affective determinants of organizational citizenship behavior. Journal of Applied Psychology, 1989, (72):157-164.

[14] Randel A. E., Galvin B. M., \& Shore L. M., et al. Inclusive leadership: Realizing positive outcomes through belongingness and being valued for uniqueness. Human Resource Management Review, 2018, 28(2):190-203.

[15] Snyder, C. R., \& Fromkin, H. L. Uniqueness: The human pursuit of difference. New York, 1980.

[16] Baumeister, R. F., \& Leary, M. R. The need to belong: Desire for interpersonal attachments as a fundamental human motivation. Psychological Bulletin, 1995, 117:497-529.

[17] Carmeli, A., Reiter-Palmon, R., \& Ziv, E. Inclusive leadership and employee involvement in creative tasks in the workplace: The mediating role of psychological safety. Creativity Research Journal, 2010, 22(3):250-260.

[18] Mor, M. E., Levin, A. Outside of the corporate mainstream and excluded from the work community: A study of diversity, job satisfaction and well-being. Community, Work \& Family, 2002, 5(2):133-157.

[19] Shore, L. M, Randel, A. E., \& Chung, B. G., et al. Inclusion and diversity in work groups: A Review and model for future research. Journal of Management, 2011, 37(4):1262-1689.

[20] Kulkarni, M. Contextual factors and help seeking behaviors of people with disabilities. Human Resource Development Review, 2012, 11(1):77-96.

[21] Bernstein, R. S., \& Bilimoria, D. Diversity perspectives and minority nonprofit board member inclusion. Equality, Diversity and Inclusion: An International Journal, 2013, 32(7):636-653.

[22] Conger, J. A., \& Kanungo, R. N. The empowerment process: Integrating theory and practice. Academy of Management Review, 1988, 13:471-482.

[23] Spreitzer, G. M. Psychological empowerment in the workplace: Dimensions, measurement, and validation. Academy of Management Journal, 1995, 38:1442-1465.
[24] Thomas, K. W., \& Velthouse, B. A. Cognitive elements of empowerment. Academy of Management Review, 1990, 15:666-681.

[25] Gist, M.E. Self-efficacy: Implications for organizational behavior and human resource management. The Academy of Management Review, 1987, 12:472-485.

[26] Deci, E. L., Connell, J. P., \& Ryan, R. M. Selfdetermination in a work organization. Journal of Applied Psychology, 1989, 74:580-590.

[27] Ashforth, B. E. The experience of powerlessness in organizations. Organizational Behavior and Human Decision Processes, 1989, 43: 207-242.

[28] Choi, S. B., Tran, T. B. H., \& Kang, S. W. Inclusive Leadership and Employee Well-Being: The Mediating Role of Person-Job Fit. Journal of Happiness Studies, 2017, 18(6):1-25.

[29] Deci, E. L., \& Ryan, R. M. A motivational approach to self: Integration in personality in Nebraska symposium on motivation, 1990: Perspectives on motivation (pp. 237-288). Lincoln, NE: University of Nebraska Press, 1991.

[30] Hollander, E. Inclusive leadership: The essential leader-follower relationship. New York: Routledge, 2009.

[31] Motowidlo, S. J., \& Van Scotter, J. R. Evidence that task performance should be distinguished from contextual performance. Journal of Applied Psychology, 1994, 79:475-480.

[32] Wayne S. J., Shore L. M. Liden R. C. Perceived organizational support and leader-member exchange: A social exchange perspective. Academy of Management Journal, 1997, 40(1):82111.

[33] Strauss G. Human relations-1968 style. Industrial Relations: A Journal of Economy and Society, 1968, 7(3):262-276.

[34] Stajkovic, A. D., \& Luthans, F. Self-efficacy and work-related performance: A meta-analysis. Psychological Bulletin, 1998, 124(2):240-261.

[35] Hackman, J. R., \& Oldham, G. R. (Eds.). Work redesign. Reading, MA: Addison-Wesley, 1980.

[36] Kanter, R. M. (Ed.). The change masters. New York: Simon and Schuster, 1983.

[37] Higgins, E. T. Beyond pleasure and pain. American Psychologist, 1997, 52: 1280-1300.

[38] Higgins, E. T. Promotion and prevention: regulatory focus as a motivational principle, 
advances in experimental psychology, 1998, 30:146.

[39] Birton, J. C. \& Joshua, S. B. Impacts of regulatory focus and institutions on innovation. Management Decision, 2018, MD-11-2016-0826.

[40] Benjamin, L.Z. \& Flynn, F. J. Leadership style and regulatory mode: value from fit. Organizational Behavior \& Human Decision Processes, 2006, 100(2):216-230.

[41] Jinping, L. Research on the influence of adjustment orientation and psychological empowerment on the creativity of knowledge workers. Tianjin: Tianjin University of Finance and Economics, 2018.

[42] Wang, J., \& Lee, A. Y. The role of regulatory focus in performance construction. Journal of Marketing Research, 2006, 43(1):28-38.

[43] Crowe, E., \& Higgins, E. T. Regulatory focus and strategic inclinations: promotion and prevention in decision-making. Organizational Behavior and Human Decision Processes, 1997, 69:117-132.

[44] Friedman, R. S., \& Forster, J. The effects of promotion and prevention cues on creativity. Journal of Personality and Social Psychology, 2001, 81:001-1013.

[45] Tumasjan, A. \& Braun, R. In the eye of the beholder: How regulatory focus and self-efficacy interact in influencing opportunity recognition. Journal of Business Venturing, 2012, 27: 622-636.

[46] Zhang X., \& Bartol, K. M. Linking empowering leadership and employee creativity: the influence of psychological empowerment, intrinsic motivation, and creative process engagement. Academy of Management Journal, 2010, 53(1):107-128.

[47] Atkinson, J. W. Motivational determinants of risk taking behavior. Psychological Review, 1957, 64: 381-390.

[48] Mumford, M. D. Managing creative people: strategies and tactics for innovation. Human Resource Management Review, 2000, 10(3):313351.

[49] Xiaohong, W. An empirical study on the relationship between transactional leadership and dynamic capabilities of collaborative innovation teams: the mediating role of adjustment orientation. Scientific progress and countermeasure, 2018,35(22):138-144.

[50]Hamstra, M. R. W., Yperen, N. W. V., \& Wisse, B., et al. Transformational-transactional leadership styles and followers' regulatory focus. Journal of Personnel Psychology, 2011, 10(4):182-186.

[51] Baron, R. M., \& Kenny, D. A. The moderatormediator variable distinction in social psychological research: Conceptual, strategic and statistical considerations. Journal of Personality and Social Psychology, 1986, 51:1173-1182.

[52] Lockwood, P. C. H., \& Jordan, Z. Kunda. Motivation by positive or negative role models: Regulatory focus determines who will best inspire us. Journal of Personality and Social Psychology, 2002, 83(4):854-864.

[53] Kenneth S. L., Chi-Sum W., \& Duanxu W., et al. Effect of supervisor-subordinate guanxi on supervisory decisions in China: an empirical investigation. Journal of Human Resource Management, 2000, 11(4):751-765.

[54] Baron, R. M., \& Kenny, D. A. The moderatormediator variable distinction in social psychological research: Conceptual, strategic and statistical considerations. Journal of Personality and Social Psychology, 1986, 51:1173-1182.

[55] Ting, S. The effect of inquiry leadership style on job performance, 2019, (6):285.

[56] Li, W. Research on the Influence of Serviceoriented Leadership on Employees' Work Performance-Journal of Nanchang Teachers College, 2019, (4):24-27.

[57] Liang, M. Path analysis of the impact of transformational leadership style and nurses' organizational commitment on job performance. China Health Service Management, 2017, 348(6):415-431.

[58] Shore, L. M, Randel, A. E., \& Chung, B. G., et al. Inclusion and diversity in work groups: A Review and model for future research. Journal of Management, 2011, 37(4):1262-1689.

[59] Kulkarni, M. Contextual factors and help seeking behaviors of people with disabilities. Human Resource Development Review, 2012, 11(1):77-96.

[60] Menon, S. T. Employee empowerment: An integrative psychological approach. Journal of Applied Psychology, 2001, 50(1):153-180.

[61] PengCheng, D., Yao, Y. The impact of inclusive leadership on employee job satisfaction-based on the mediating effect of psychological ownership. Corporate Economy, 2017, (8):96-103. 